\title{
Ab Initio Modeling of Graphene Functionalized with Boron and Nitrogen
}

\author{
M. Woińska, K. Milowska And J.A. MAJEWSKI
}

Institute of Theoretical Physics, Faculty of Physics, University of Warsaw, Hoża 69, 00-681 Warsaw, Poland

We investigate theoretically the electronic properties of graphene functionalized with nitrogen and boron atoms substituted into the graphene monolayer. Our study is based on the ab initio calculations in the framework of the density functional theory. We predict the dependence of the energy band gap, binding energy per atom, and the shift of the Fermi level on the concentration of dopants. Moreover, we examine the influence of the distribution of $\mathrm{B} / \mathrm{N}$ atoms on the specified properties.

PACS: 73.22.Pr, 61.48.Gh, 81.05.ue

\section{Introduction}

Graphene is a material with unique electronic and mechanical properties. Due to its hexagonal symmetry its valence and conduction band are characterized by a zero band gap and linear dispersion relation in the $K$ point. It also results in electron mobility extremely high in comparison with semiconductors [1], which in connection with excellent mechanical properties renders graphene an ideal candidate for applications in flexible electronics. However, the ability of generating controllable band gap is a prerequisite for electronics based on graphene. Therefore, an effective functionalization that would open the zero energy band gap in pristine graphene without significant deterioration of the remaining advantageous properties is searched for and practically any thinkable way of reaching this goal is currently investigated.

There exist three general methods of opening the band gap in graphene: application of external electric field, cutting nanoribbons out of graphene planes, and graphene functionalization with various molecules and dopants. In this paper, we study the effects of the third technique, namely the doping of the graphene layer with boron and nitrogen [2]. These elements, standing nearby carbon in the periodic table, should also act as electron acceptors (B) and donors (N), allowing for fabrication of contemporary devices in the manner of the CMOS silicon technology. Here we report new results of our theoretical studies of electronic properties of graphene doped with boron and nitrogen atoms.

\section{Computational approach}

Our studies of functionalized graphene layer are based on $a b$ initio calculations within the framework of the spin polarized density functional theory (DFT) [3, 4]. Generalized gradient approximation of the exchange-correlation functional in the Perdew-Burke-Ernzerhof (PBE) parameterization has been applied [5]. We employed the SIESTA package [6, 7], involving the usage of the periodic boundary conditions and supercell geometry, with graphene layers separated by a distance large enough to eliminate any spurious interactions. Valence electrons have been represented with double zeta basis sets of orbitals localized on atoms; influence of core electrons was covered within pseudopotential formalism. Norm-conserving Troullier-Martins nonlocal pseudopotentials [8] in the Kleinman-Bylander [9] form have been used to account for the electron-ion interactions. The energy cutoff determining the density of the utilized real space grid was set to $800 \mathrm{Ry}$, which guarantees excellent convergence and accuracy. Structural optimization has been conducted employing the conjugate gradient algorithm to achieve residual forces acting on the atoms lower than $0.001 \mathrm{eV} / \AA$

To investigate the dependence of the doping induced band gap, and stability of the doped structures on the concentration of the dopants, we consider first $2 \times 2$, $3 \times 3,4 \times 4,5 \times 5$ and $6 \times 6$ graphene supercells with one carbon atom substituted with boron or nitrogen atom. This corresponds, respectively, to dopant concentrations of $12.5 \%, 5.56 \%, 3.13 \%, 2 \%$, and $1.39 \%$. In all cases we optimize geometry of the doped structures and further calculate the binding energy per atom, energy band gap, shift of the Fermi level with respect to the top of the valence band (bottom of the conduction band) for B $(\mathrm{N})$. However, for a given doping concentration, the dopant atoms can be distributed in many ways in the graphene supercell. To study the effect of dopant ordering on the binding energy and the magnitude of the energy gap we introduce a parameter measuring the level of clustering (further called clustering parameter $C$ ) of the considered dopant configurations. This parameter is in general linearly proportional to the sum of $n-1$ shortest nonequivalent distances between $\mathrm{B} / \mathrm{N}$ atoms (where $n$ is the number of $\mathrm{B} / \mathrm{N}$ atoms in a supercell) and it is normalized to reach a value of -1 for maximal and 1 for minimal clustering in a system. The clustering parameter $C$ is given by the following formula:

$$
C=\frac{2 \Sigma-\Sigma_{\max }-\Sigma_{\min }}{\Sigma_{\min }-\Sigma_{\max }},
$$

where $\Sigma$ is the sum of $n-1$ shortest nonequivalent distances in the system, whereas $\Sigma_{\max }$ and $\Sigma_{\min }$ are maximal and minimal value that $\Sigma$ reaches for a given $n$. The clustering parameter $C$ for $n=2$ is the most negative when $\mathrm{B} / \mathrm{N}$ atoms are linked by a chemical bond (i.e., 
they are the nearest neighbors) and the most positive, if they are in the largest possible distance from each other.

\section{Results and discussion}

The dependence of the energy band gap on concentration of $\mathrm{B} / \mathrm{N}$ atoms investigated by means of substituting one carbon atom in a $2 \times 2,3 \times 3,4 \times 4,5 \times 5$ and $6 \times 6$ graphene supercell (with corresponding concentrations: $12.5 \%, 5.56 \%, 3.13 \%, 2 \%$ and $1.39 \%)$ is presented in Fig. 1. The band gaps for a given concentration are very similar for boron and nitrogen dopants. For $3 \times 3$ and $6 \times 6$ supercells, we do not observe band gap opening, which is consistent with the phenomenon of zero band gap for supercells with dimensions being multiples of three graphene lattice constants described earlier by García-Lastra [10]. For the other supercells, we observe that the energy band gap increases generally with concentration. For example, the energy gap increases from $0.13 \mathrm{eV}$ to $0.54 \mathrm{eV}$ for one $\mathrm{B}$ in $5 \times 5$ (concentration $2 \%$ ) and $2 \times 2$ (concentration $12.5 \%$ ) supercells, respectively. Thus, it may be concluded that functionalization (doping) of graphene with boron or nitrogen allows for opening the band gap in graphene. However, in ordered structures considered in these studies, the certain built in periodicity can suppress the effect of the band gap opening. Therefore, it could hinder the comparison with experiments which definitely deal with systems exhibiting truly random distribution of dopants in the graphene matrix.

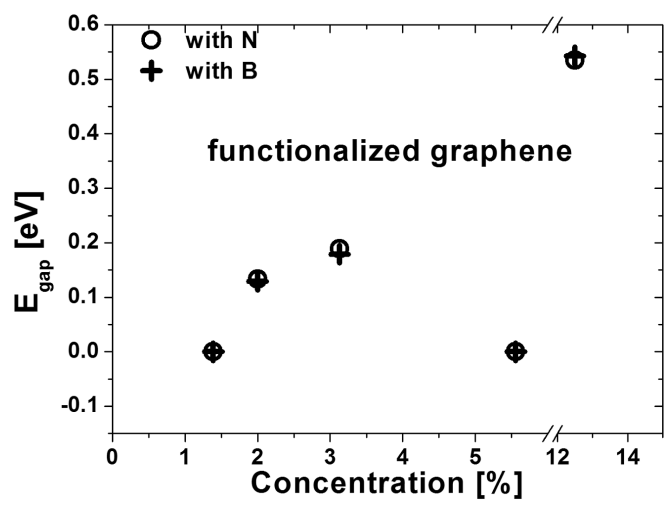

Fig. 1. Band gap energies for $2 \times 2,3 \times 3,4 \times 4,5 \times 5$, and $6 \times 6$ graphene supercells containing one $\mathrm{B} / \mathrm{N}$ atom per supercell, i.e., for dopant concentration of $12.5 \%$, $5.56 \%, 3.13 \%, 2 \%$, and $1.39 \%$, respectively.

For a given doping concentration, the $\mathrm{B}(\mathrm{N})$ atoms can be substituted into the carbon sites in many configurations. To investigate this phenomenon, we consider all 11 symmetrically nonequivalent configurations of two $\mathrm{B}(\mathrm{N})$ atoms in $5 \times 5$ graphene supercell (it corresponds to doping concentration equal to $4 \%$ ) and find optimized geometry of the system. Each dopant configuration can be characterized by the clustering parameter $C$. In Fig. 2, the geometries for two extreme values of clustering parameter $C$ are depicted. Also the isolines of electron density in the graphene plane are presented there. We observe regions of electron density depletion around the positions of boron atoms, as well as increase of electron density in the vicinity of nitrogen atoms. Substituent atoms influence only the electron density around those carbon atoms with which they are linked by a chemical bond. Electronic charge around the second and further neighbors remains unchanged.
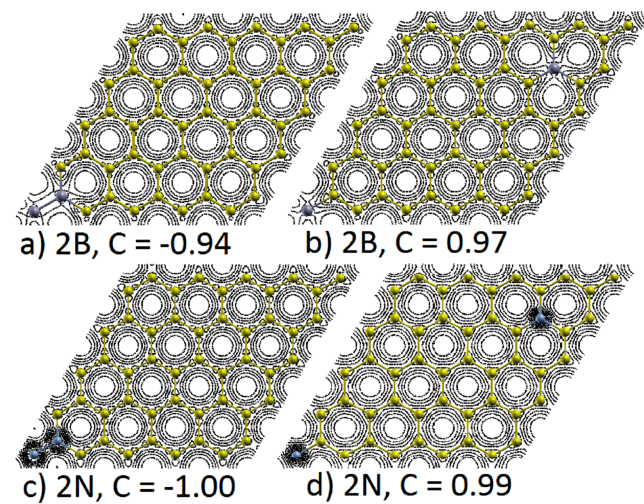

Fig. 2. Electron density in the $5 \times 5$ graphene supercell with two dopant atoms; (a) and (b) for boron, and (c) and (d) for nitrogen. Parts (a) and (c) describe the situation where dopant atoms are in the closest possible position, whereas parts (b) and (d) the situation where dopants are in the largest possible distance from each other. This is illustrated by two extreme values of the clustering parameter $C$. Let us note the depletion of electron density in the vicinity of B atoms [parts (a) and (b)] and increase of electron density around the positions of $\mathrm{N}$ atoms [parts (c) and (d)].

For each fully relaxed configuration, we calculate binding energies per atom, the band gap energy, and the shift of the Fermi level from the bottom of the conduction band (CBB) for N and the top of the valence band (VBT) for $B$. The calculated quantities, together with the values of the clustering parameter $C$, for each of the 11 configurations are presented in Table I for boron, and in Table II for nitrogen. It can be noticed that clustering parameters are quite similar for the same configurations of atoms in the case of $\mathrm{B}$ and $\mathrm{N}$ doping, which is the consequence of the fact that for such small concentrations substitution does not distort geometry of graphene layer significantly. All the investigated structures are stable, which can be confirmed by their meaningfully negative binding energies. Stability is insignificantly higher in the case of B-functionalized graphene, with the energy difference of about $0.05 \mathrm{eV}$ per atom in comparison with $\mathrm{N}$-functionalized structures.

As far as the width of the band gap is concerned, in the majority of the cases it has much the same values for $\mathrm{B}$ and $\mathrm{N}$ atoms being in the same configuration. Moreover, the band gap for one atom per $5 \times 5$ supercell and its averaged value for 11 configurations of two $\mathrm{B}$ and two 
$\mathrm{N}$ atoms in $5 \times 5$ supercell are roughly equal. One can notice that band gap widths vary from 0 to slightly less than $0.3 \mathrm{eV}$ and no particular influence of clustering parameter on these values can be affirmed. To have a better insight into this problem, further calculations for higher concentrations should be supportive. We have started such studies within the empirical tight-binding method, and the results will be published elsewhere. Of course, doping of graphene layer with boron (acceptor) or nitrogen (donor) changes the position of the Fermi level. The Fermi level shift with respect to VBT (for B) or CBB (for $\mathrm{N}$ ) in the doped graphene is quite similar for the system doped with $\mathrm{B}$ and $\mathrm{N}$ in identical configuration, being often slightly higher for boron.

TABLE I

The calculated values of band gap energy $\left(E_{\mathrm{gap}}\right)$, Fermi level shift with respect to the top of the valence band $\left(E_{\mathrm{VBT}}-\right.$ $\left.E_{\text {Fermi }}\right)$, binding energy per atom $\left(E_{\text {bond }}\right)$ and clustering parameter $(C)$ for 11 various configurations of two B atoms in $5 \times 5$ graphene supercell. Values for maximum and minimum $E_{\text {gap }}$ are also detailed along with values averaged over all the configurations. On the right hand side the results for one B in $5 \times 5$ supercell are given.

\begin{tabular}{|c|c|c|c|c|c|c|c|c|c|c|c|c|c|c|c|}
\hline \multirow{2}{*}{$\begin{array}{c}\text { Energy } \\
{[\mathrm{eV}]}\end{array}$} & \multicolumn{14}{|c|}{ Configuration of two $\mathrm{B}$} & \multirow{2}{*}{ One B } \\
\hline & 1 & 2 & 3 & 4 & 5 & 6 & 7 & \begin{tabular}{|l|}
8 \\
\end{tabular} & 9 & 10 & 11 & $E_{\text {gap,min }}$ & $E_{\text {gap, } \max }$ & Mean & \\
\hline$E_{\text {gap }}$ & 0.27 & 0.23 & 0.00 & 0.01 & 0.01 & 0.24 & 0.23 & 0.00 & 0.00 & 0.08 & 0.00 & 0.00 & 0.27 & 0.10 & 0.13 \\
\hline$E_{\mathrm{VBT}}-E_{\mathrm{Fermi}}$ & 0.78 & 0.90 & 0.89 & 1.03 & 0.88 & 0.81 & 0.84 & 1.02 & 0.91 & 0.88 & 0.67 & 0.67 & 0.78 & 0.87 & 0.69 \\
\hline$E_{\text {bond }}$ & -8.54 & -8.55 & -8.53 & -8.55 & -8.55 & -8.55 & -8.55 & -8.55 & -8.55 & -8.55 & -8.55 & -8.55 & -8.54 & -8.55 & -8.60 \\
\hline$C$ & -0.62 & 0.25 & -0.94 & -0.16 & 0.67 & 0.01 & 0.78 & 0.52 & -0.49 & 0.31 & 0.97 & 0.97 & -0.62 & - & - \\
\hline
\end{tabular}

TABLE II

The calculated values of band gap energy $\left(E_{\text {gap }}\right)$, Fermi level shift with respect to the bottom of the conduction band $\left(E_{\mathrm{CBB}}-E_{\mathrm{Fermi}}\right)$, binding energy per atom $\left(E_{\mathrm{bond}}\right)$ and clustering parameter $(C)$ for 11 various configurations of two $\mathrm{N}$ atoms in $5 \times 5$ graphene supercell. Values for maximum and minimum $E_{\text {gap }}$ are also detailed along with values averaged over all the configurations. On the right hand side the results for one $\mathrm{N}$ in $5 \times 5$ supercell are given.

\begin{tabular}{|c|c|c|c|c|c|c|c|c|c|c|c|c|c|c|c|}
\hline \multirow{2}{*}{$\begin{array}{c}\text { Energy } \\
{[\mathrm{eV}]}\end{array}$} & \multicolumn{14}{|c|}{ Configurations of two $\mathrm{N}$} & \multirow{2}{*}{ One $\mathrm{N}$} \\
\hline & 1 & 2 & 3 & 4 & 5 & 6 & 7 & 8 & 9 & 10 & 11 & $E_{\text {gap,min }}$ & $E_{\text {gap, max }}$ & Mean & \\
\hline$E_{\text {gap }}$ & 0.28 & 0.24 & 0.15 & 0.00 & 0.01 & 0.25 & 0.24 & 0.00 & 0.01 & 0.04 & 0.01 & 0.00 & 0.28 & 0.10 & 0.13 \\
\hline$E_{\mathrm{Fermi}}-E_{\mathrm{CBB}}$ & 0.77 & 0.86 & 0.81 & 0.99 & 0.99 & 0.85 & 0.82 & 0.99 & 0.99 & 0.93 & 0.92 & 0.99 & 0.77 & 0.90 & 0.66 \\
\hline$E_{\text {bond }}$ & -8.50 & -8.50 & -8.48 & -8.50 & -8.51 & -8.50 & -8.50 & -8.51 & -8.51 & -8.51 & -8.51 & -8.50 & -8.50 & -8.50 & -8.58 \\
\hline$C$ & -0.65 & 0.23 & -1.00 & -0.19 & 0.67 & -0.01 & 0.78 & 0.49 & -0.50 & 0.30 & 0.99 & -0.19 & -0.65 & - & - \\
\hline
\end{tabular}

\section{Conclusions}

In this paper we report the results of $a b$ initio calculations for graphene layer functionalized with boron and nitrogen atoms. We investigate structures with one $\mathrm{B}(\mathrm{N})$ atom in graphene supercells with various dimensions and structures with two B $(\mathrm{N})$ atoms in a $5 \times 5$ supercell. All the structures are stable with slightly more negative binding energies in the case of graphene doped with B. We conclude that the functionalization caused band gap opening in graphene is strongly dependent on a particular configuration of atoms, at least in case of considered here periodic structures. Furthermore, the energies of the band gap turn out to be very similar for analogical structures functionalized with boron and nitrogen.

\section{Acknowledgments}

This work has been supported by the European Founds for Regional Development within the SiCMAT project (contact No. UDA-POIG.01.03.01-14-155/09). This research was supported in part by PL-Grid Infrastructure. Some of the computations were performed using a cluster of ICM Interdisciplinary Centre for Mathematical and Computational Modeling (grant No. G47-5 and G47-16, University of Warsaw).

\section{References}

[1] A.K. Geim, K.S. Novoselov, Nat. Mater. 6, 183 (2007).

[2] L.S. Panchakarla, K.S. Subrahmanyam, S.K. Saha, A. Govindaraj, H.R. Krishnamurthy, U.V. Waghmare, C.N.R. Rao, Adv. Mater. 21, 4726 (2009).

[3] P. Hohenberg, W. Kohn, Phys. Rev. 136, 864 (1964).

[4] W. Kohn, L.J. Sham, Phys. Rev. 140, A1133 (1965).

[5] J.P. Perdew, K. Burke, M. Ernzerhof, Phys. Rev. Lett. 77, 3865 (1996).

[6] D. Sanchez-Portal, P. Ordejon, E. Artacho, J.M. Soler, Int. J. Quantum Chem. 65, 453 (1997).

[7] J.M. Soler, E. Artacho, J. Gale, A. Garcia, J. Junquera, P. Ordejon, D. Sanchez-Portal, J. Phys., Condens. Matter 14, 2745 (2002).

[8] N. Troullier, J.L. Martins, Phys. Rev. B 43, 1993 (1991).

[9] L. Kleinman, D.M. Bylander, Phys. Rev. Lett. 48, 1425 (1982).

[10] J.M. García-Lastra, Phys. Rev. B 82, 235418 (2010). 